\title{
The Relationship between the Perception and Performance of Headache Management among School Nurses
}

\author{
Shinyoung Park1), Youngil Rho2) Misun Jang3)
}

\begin{abstract}
This study was conducted to investigate the relationship between school nurses's perception level and performance level about headache management for children and adolescents with headache in elementary school, middle school, and high school. The subjects of this study were 250 school nurses working in elementary, middle, and high schools in $\mathrm{G}$ metropolitan city, who had a nurse's license and participated in the training course of the $\mathrm{G}$ metropolitan office of education. After excluding survey responses with incomplete information, there were 176 participants in total. For the data collection, a structured questionnaire was used, and the collected data were performed using the SPSS 22.0 for Windows program, average analysis, frequency analysis, ANOVA, Pearson's correlation, and multiple regression analysis. Participants were 176 school nurses; The average scores for perception level and performance level of headache management were $3.88 \pm 0.33$ (range 1-5) and $3.79 \pm 0.40$ (range 1-5), respectively. School nurses's perception level and performance level of headache management showed a statistically significant correlation ( $\mathrm{r}=0.50, \mathrm{p}<0.001)$. As a result of multiple regression analysis, it can be seen that the level of perception of headache management by school nurses affects the level of performance of headache management by school nurses, and this explanatory power was $24.5 \%$. These results showed that the performance levels of school nurses on headache management for students with headache were lower than the perception levels of them, and indicated that the performance levels of school nurses on headache management for students with headache increased as the perception levels of them increased. Therefore, it suggests to develop various training programs for effective headache management of school nurses and to develop programs and standardized guidelines to manage health problems, including headaches among children and adolescents.
\end{abstract}

Keywords: School Nursing, Headache, Perception, Performance

\section{Introduction}

School nurses are in charge of the student's health issues, providing primary health care

Received(November 21, 2019), Review Result(1st: December 26, 2019, 2nd: February 12, 2020), Accepted(April 25, 2020)

1) (Professor) 61453 College of Chosun Nursing, Gwangju, Korea email: cylove39@naver.com

2) (Professor, Corresponding Author) 61453 Department of Pediatrics, School of Medicine, Chosun University, Gwangju, Korea

email: ryoung@chosun.ac.kr

3) (Nurse) 58141 Fourfree, Hwasun-gun, Jeollanam-do, Republic of Korea.

email: sangkm3507@nate.com 
services such as early detection and diagnosis of possible health-related issues in the students, and preventing potential progression or worsening of these issues[1-3]. Due to the recent changes in the school culture there is an increasing number of students exhibiting health-related issues and visiting the school nurse. More specifically, there are an increasing number of students exhibiting neurological symptoms, especially headaches[3-5]. There are multiple symptoms frequently observed in the students including headache (5-30\%), abdominal pain (10-38\%), and other symptoms (i.e. fatigue, dizziness, nausea, and pain in their limb)[6][7], which are the key symptoms in Korean students for visiting the school nurse[8].

In Korea, $29.1 \%$ of the students experience repetitive or persistent headache, and the frequency is remarkably higher in high school students compared to elementary school students. Migraine accounts for $8.7 \%$ of the headache in students on average, and is more frequently observed in females than males and seniors than juniors[9]. Repetitive and persistent headache, in severe cases, can prevent the students from attending their class regularly and thereby bring negative impact on their academic life, cause troubles in the relationships between parents and friends, and reduce their quality of life[10][11]. Moreover, although headache at younger age may be temporary and tolerable, it may be associated with fundamental pathological status in the future, and thus it should be carefully examined[12].

Since headache may hinder academic success in the students and bring negative impact on their school life, school nurses should be able to appropriately and effectively manage the condition. A recent study assessing the management of headache in the students by school nurses demonstrated that school nurses lacked general knowledge despite having an important role in the management of headache in the students. In particular, these lacked knowledge of evaluation methods despite asking many questions to the students, and provided care without proper guideline for headache management[13]. Nonetheless, there are no previous studies that assessed perception and performance of headache management in the school nurses (i.e. most recent trend and importance of headache management).

This study therefore aims to assess perception and performance of headache management in the school nurses, as well as the correlation between perception and performance, in order to efficiently manage headaches in the students - which is one of the key health-related issues - by assisting with establishing standardized guideline of headache management for the school nurses.

\section{Methods}




\subsection{Sample}

The participants of this study included 250 school nurses from elementary, middle, and high schools in $G$ metropolitan city who participated in the training lecture provided by the $G$ metropolitan office of education. 250 school nurses came from different schools (i.e. only one school nurse per school), and had nurse license. The study was performed after providing explanation for the purpose and content of the study, and obtaining voluntary consent from the participants. After excluding survey responses with incomplete information, there were 176 participants in total.

\subsection{Instrument}

This study is a short descriptive study based on a survey questionnaire that includes 7 questions on general characteristics, 10 questions on work-related characteristics, 11 questions on the perception of headache management, and 16 questions on the performance of headache management. The participants spent $\sim 10$ minutes in average to complete the survey.

The general characteristics of school nurses included age, work experience (years), previous workplace, perception of the need for clinical experience, education level, and current workplace. For work-related characteristics, the following questions were included: most frequently performed task, most frequently observed health issues in the students, health-related issues that are most difficult to manage, the proportion of students with headache and their causes, confidence in headache management, primary care for headache, participation in training lectures for headache management in the recent 3 years, and willingness to participate in the training lecture for headache management in the future.

For the evaluation of the perception levels of school nurses on headache management for students with headache, the survey developed by Jeon et al[7] was modified and used in the research. For each question, participants were asked to provide response on a 5-point Likert scale ranging from 1 (strongly disagree) to 5 (strongly agree). The scores for reverse questions (2 and 5) were appropriately converted prior to calculating the average score. Higher score indicated greater perception of headache management, and the reliability of this tool was Cronbach's $a=0.684$.

Similarly, the performance of headache management was assessed using a modified version of the survey questionnaire developed by Jeon et al.[7]. Each question was on a 5-point Likert scale ranging from 1 (strongly disagree) to 5 (strongly agree). Higher score indicated greater 
performance of headache management, and the reliability of this tool was Cronbach's $a=0.825$.

\subsection{Data Analysis}

Statistical analyses in this study were performed using SPSS 22.0 for Windows (SPSS Ins., Chicago, IL, USA). Descriptive statistics of the general characteristics, work-related characteristics, perception of headache management, and performance of headache management were represented using the real numbers, percentages, mean, and standard deviation values. Furthermore, the reliability of the tools used for assessment of perception and performance of headache management was evaluated using Cronbach's a. To understand the difference in the perception and performance of headache management based on the general characteristics, analysis of variance (ANOVA) was performed. In addition, to assess the correlation between the perception and performance of headache management, Pearson correlation analysis was performed. Lastly, multiple regression analysis was performed to identify the factors that affect the performance of headache management in the school nurses. Analysis outcomes with $\mathrm{P}<0.05$ were considered statistically significant.

This study was approved by the institutional review board of Chosun University Hospital (IRB no. 2017-12-005-001).

\section{Results}

\subsection{General Characteristics of the Participants}

The mean age of the participants was $42.47 \pm 8.30$, and the mean work experience of them was 12.1 \pm 9.27 years. For the previous workplace, most of the participants came from tertiary health care centers (89 participants, 50.6\%) followed by secondary centers (36 participants, 20.5\%). The majority of participants (137 participants, 77.8\%) thought that previous clinical experience is necessary, and most of the participants were 4-year program university graduates (112 participants, 63.6\%). There were 100 participants (56.8\%) from elementary school and 50 participants $(28.4 \%)$ from middle school. Remarkable number of participants (145 participants, $82.4 \%$ responded that they have average level of confidence for headache management, but vast majority of the participants (154 participants, 87.5\%) had not participated in training lectures for headache management in the past 3 years. Moreover, there was no significant difference between perception and performance of headache management according to the 
[Table 1] Recognition and Performance of Headache Management according to General Characteristics of School Nurses

$(\mathrm{N}=176)$

\begin{tabular}{|c|c|c|c|c|c|}
\hline \multirow[t]{2}{*}{ Characteristics and classification } & \multirow[t]{2}{*}{$\mathrm{n}(\%)$ or $\mathrm{M} \pm \mathrm{SD}$} & \multicolumn{2}{|c|}{ recognition } & \multicolumn{2}{|c|}{ Performance } \\
\hline & & $\mathrm{M} \pm \mathrm{SD}$ & $\mathrm{t}$ or $\mathrm{F}(p)$ & $\mathrm{M} \pm \mathrm{SD}$ & $\mathrm{t}$ or $\mathrm{F}(p)$ \\
\hline Age (yrs) & $42.47 \pm 8.30$ & & & & \\
\hline Clinical career & $12.1 \pm 9.27$ & & & & \\
\hline \multicolumn{6}{|c|}{ Previous work place } \\
\hline Tertiary medical institution & $89(50.6)$ & $3.88 \pm 0.32$ & $0.56(0.69)$ & $3.79 \pm 0.43$ & $1.23(0.30)$ \\
\hline Secondary medical institution & $36(20.5)$ & $3.90 \pm 0.36$ & & $3.81 \pm 0.40$ & \\
\hline Public Health & $2(1.1)$ & $3.59 \pm 0.19$ & & $3.19 \pm 0.18$ & \\
\hline other & $25(14.2)$ & $3.91 \pm 0.33$ & & $3.79 \pm 0.34$ & \\
\hline none & 23(13.1) & $3.84 \pm 0.28$ & & $3.83 \pm 0.34$ & \\
\hline \multicolumn{6}{|c|}{ Clinical experience } \\
\hline Not needed & $5(2.8)$ & $3.84 \pm 0.25$ & $0.63(0.53)$ & $3.80 \pm 0.78$ & $0.01(0.99)$ \\
\hline Usually & $33(18.8)$ & $3.83 \pm 0.29$ & & $3.79 \pm 0.42$ & \\
\hline Needed & $137(77.8)$ & $3.89 \pm 0.33$ & & $3.79 \pm 0.40$ & \\
\hline \multicolumn{6}{|c|}{ Education } \\
\hline College & $25(14.2)$ & $3.88 \pm 0.34$ & $0.04(0.96)$ & $3.85 \pm 0.39$ & $0.40(0.67)$ \\
\hline University & $112(63.6)$ & $3.88 \pm 0.32$ & & $3.77 \pm 0.42$ & \\
\hline Graduate school & $38(21.6)$ & $3.89 \pm 0.33$ & & $3.80 \pm 0.34$ & \\
\hline \multicolumn{6}{|c|}{ Current workplace } \\
\hline Elementary & $100(56.8)$ & $3.88 \pm 0.32$ & $0.10(0.91)$ & $3.79 \pm 0.37$ & $0.01(0.99)$ \\
\hline Middle & $50(28.4)$ & $3.88 \pm 0.33$ & & $3.79 \pm 0.42$ & \\
\hline High & $26(14.8)$ & $3.91 \pm 0.38$ & & $3.78 \pm 0.47$ & \\
\hline \multicolumn{6}{|c|}{ Confidence in headache management } \\
\hline Confidence & $17(9.7)$ & $3.90 \pm 0.36$ & $0.42(0.66)$ & $3.80 \pm 0.43$ & $0.54(0.58)$ \\
\hline Usually & $145(82.4)$ & $3.87 \pm 0.33$ & & $3.80 \pm 0.39$ & \\
\hline Can not & $14(8.0)$ & $3.95 \pm 0.29$ & & $3.68 \pm 0.43$ & \\
\hline \multicolumn{6}{|c|}{ Participate in the practical training program within 3 years } \\
\hline Yes & $22(22.5)$ & $3.97 \pm 0.35$ & $-1.29(0.20)$ & $3.84 \pm 0.37$ & $-0.60(0.55)$ \\
\hline None & 154(87.5) & $3.87 \pm 0.32$ & & $3.78 \pm 0.40$ & \\
\hline
\end{tabular}

\subsection{Work-related Characteristics of the Participants}

The most frequently performed task by school nurses was primary emergency care and management of health-related issues $(82.4 \%)$. The most frequently observed health issues in the students leading to school nurse visit was wounds $(34.1 \%)$, followed by abdominal pain $(30.7 \%)$, headache $(17.6 \%)$, and sprain or fracture $(13.1 \%)$. For health-related issues that are most difficult to manage, the majority of participants responded abdominal pain (52.3\%) and headache $(29.5 \%)$, with $21.2 \pm 13.48 \%$ being the daily mean proportion \pm standard deviation of the students who visit school nurses due to headache. The key causes of headache in the 
students were irregular sleep pattern and empty stomach $(36.9 \%)$, as well as relationship issues $(30.1 \%)$. Only $9.7 \%$ of the participants responded that they are confident in headache management, and the primary care for headache management were relaxation on the bed $(42.6 \%)$ and observation (30.1\%). For efficient headache management, the participants responded that standardized guideline for headache management $(49.4 \%)$ as well as training lecture $(43.8 \%)$ are needed. Only $12.5 \%$ of the participants had attended practical training lecture program for headache management in the past 3 years, and $75.0 \%$ of the participants were interested in participating in the future training lectures [Table 2].

[Table 2] Work Related Characteristics of the Subject

$(\mathrm{N}=176)$

\begin{tabular}{|c|c|}
\hline Characteristics & $\mathrm{n}(\%)$ \\
\hline \multicolumn{2}{|l|}{ The highest proportion of school health work } \\
\hline Health education & $28(15.9)$ \\
\hline First Aid and Health Problem Management & $145(82.4)$ \\
\hline Environmental Management & $2(1.1)$ \\
\hline Employee Healthcare & $1(0.6)$ \\
\hline \multicolumn{2}{|l|}{ Most health problems visiting the nursing home } \\
\hline Wound & $60(34.1)$ \\
\hline Colic & $54(30.7)$ \\
\hline Headache & $31(17.6)$ \\
\hline Cold, chills & $4(2.3)$ \\
\hline Sprains and fractures & $23(13.1)$ \\
\hline Others & $4(2.3)$ \\
\hline \multicolumn{2}{|l|}{ The most unmanageable problem } \\
\hline Wound & $3(1.7)$ \\
\hline Colic & $92(52.3)$ \\
\hline Headache & $52(29.5)$ \\
\hline Sprains and fractures & $16(9.1)$ \\
\hline Others & $13(7.4)$ \\
\hline $\begin{array}{l}\text { Percentage of students who complain of headache among visiting students } \\
\text { Causes of student headaches }\end{array}$ & $21.2 \pm 13.5 \%$ \\
\hline Basic lesion & $14(8.0)$ \\
\hline Irregular sleep habits and fasting & $65(36.9)$ \\
\hline Relationship problem & $53(30.1)$ \\
\hline Academic problems & $41(23.3)$ \\
\hline \multicolumn{2}{|l|}{ Confidence in Headache Management } \\
\hline With confidence & $17(9.7)$ \\
\hline Usually & $145(82.4)$ \\
\hline Can not & $14(8.0)$ \\
\hline
\end{tabular}

Primary management of headache 
Observe

Others

A method for effective headache management

Job training program

Standardized headache management guidelines

Participation in the field training program within the last 3 years

$$
\begin{aligned}
& \text { None } \\
& \text { Yes }
\end{aligned}
$$

Intent to participate in the field work program

$$
\text { No }
$$

Usually

Yes

\subsection{Perception and Performance of Headache Management in the Participants}

The mean score \pm standard deviation for perception of headache management in the school nurses was $3.88 \pm 0.33$, and the following questions had highest and lowest scores respectively: 'it is important to identify whether or not the headache symptom is causing an emergency' $(4.53 \pm 0.65)$ and 'it is easy to manage headaches as its causes are relatively simple' $(2.05 \pm 0.84)$. The mean score \pm standard deviation for performance of headache management was $3.79 \pm 0.40$, and the following questions had highest and lowest scores respectively: 'students with accompanying symptoms of fever and vomiting should be taken to nearby hospital for further treatment' $(4.55 \pm 0.57)$ and 'information regarding the headache in the student is shared with the clinician treating the student' $(2.61 \pm 0.93)$. In general, the perception of headache management was greater than the performance in this cohort of school nurses [Table 3].

[Table 3] Descriptive Statistics on the Perception and Performance of Headache Management

$(\mathrm{N}=176)$

\begin{tabular}{lc}
\hline Categories & $\mathrm{M} \pm \mathrm{SD}$ \\
\hline Perception of headache management & $3.88 \pm 0.33$ \\
1. Adequate and professional level knowledge is required for headache management. & $4.27 \pm 0.52$ \\
2. It is easy to manage headaches as its causes are relatively simple. & $2.05 \pm 0.84$ \\
3. Expert health assessment skills are needed for headache management. & $4.24 \pm 0.58$
\end{tabular}


4. Knowledge on the medication (mechanism, effect, side-effects) for headache is necessary. $\quad 4.22 \pm 0.72$

5. Headache can be sufficiently managed based on my own experience.

6. It is important to identify whether or not the headache symptom is causing an emergency.

$4.53 \pm 0.65$

7. For the management of headache in the student, information should be shared among the

$4.31 \pm 0.58$ teacher, the parents, and the local healthcare facility.

8. Proper management of headache can assist the students to adapt to school life.

$4.38 \pm 0.54$

9. Headache management accounts for a large proportion of the tasks for school nurses.

$3.73 \pm 0.77$

10. For effective headache management, a standardized guideline for headache management is

$4.26 \pm 0.59$ necessary.

11. Newest knowledge and information should be acquired for proper and efficient $4.40 \pm 0.54$ management of headache.

Performance of headache management

$3.79 \pm 0.40$

1. Medication and other materials for headache management are stocked.

$3.51 \pm 0.68$

2. Medical examination via interview is the first step for the students with headache.

$4.43 \pm 0.57$

3. Inquire the student with headache for possible injuries to the head.

$4.03 \pm 0.80$

4. Record the medical examination outcomes via interview in a written format.

$4.05 \pm 0.75$

5. Record all medical history, symptoms, treatment, and health conditions in detail.

$3.45 \pm 0.92$

6. Provide medication (if needed) after determination of the cause of headache

$3.98 \pm 0.65$

7. Speak to the parents regarding the student's headache

$3.30 \pm 0.91$

8. Share the information to the clinician regarding the student's headache

$2.61 \pm 0.93$

9. Speak to the teacher regarding the student's headache

$3.88 \pm 0.76$

10. Selectively diagnose the student's headache, receive treatment from clinician if necessary

$4.06 \pm 0.69$

11. Students with accompanying symptoms (fever, vomiting) should be treated at a medical $4.55 \pm 0.57$ center or hospital.

12. Even if the student does not have other related symptoms, s/he should be directed to $4.32 \pm 0.68$ hospital for further examination if the headache persists.

13. Students with headache should be rested in the school health room at his/her will

14. Consistent treatment is provided for management of headache patients.

15. Reduction in the symptom of headache is assessed after treatment.

16. A standardized guideline is used for management of headache.

$3.28 \pm 0.87$

\subsection{Relationship between the Perception and Performance of Headache Management in the Participants}

There was a significant positive correlation between the perception and performance of headache management in the school nurses, demonstrating greater level of performance with greater level of perception $(\mathrm{r}=0.50, p<0.001)$ [Table 4]. 
[Table 4] Correlation between the Perception and the Performance of Headache Management in School

Nurses

$(\mathrm{N}=176)$

\begin{tabular}{ccc}
\hline Categories & Perception & Performance \\
\cline { 2 - 3 } & $\mathrm{r}(p)$ & $\mathrm{r}(p)$ \\
\hline Perception & 1 & \\
Performance & $0.50(<.001)$ & 1 \\
\hline
\end{tabular}

\subsection{Factors Affecting the Performance of Headache Management}

Multiple linear regression analysis on the perception of headache management has been performed to identify the factors affecting the performance of headache management in the school nurses, and the outcome was statistically significant ( $F=57.90, p<0.001)$. Modified coefficient of determination (R2) that shows the explanatory power of the model was 0.245 [Table 5].

[Table 5] Factors Affecting the Performance of Headache Management in the School Nurses

$(\mathrm{N}=176)$

\begin{tabular}{cccccc}
\hline & Categories & B & $\beta$ & $\mathrm{t}$ & $p$ \\
\hline Perception & of headache management & .611 & .500 & 7.61 & $<.001$
\end{tabular}

$\mathrm{R}^{2}=.250$, Adj. $\mathrm{R}^{2}=.245, \mathrm{~F}=57.90, p<.001{ }^{*}$ Durbin-Watson $=1.871$; independence of residuals satisfied

\section{Discussion}

With changes in student and school culture, several health-related issues have been arising, causing the increase in the number of students visiting the school nurse - especially due to neurological symptoms (i.e. headache)[3-5]. School nurses who provide primary health care for the local community (students in general) should be able to manage the key health issues in the students effectively, but lack of standardized guidelines for management of different health-related issues in the students make it difficult for the school nurses to provide appropriate management for diverse symptoms[7].

Adolescent and teenage students exhibit different physical symptoms, and most frequently observed symptoms include headache (5-30\%), abdominal pain (10-38\%), fatigue, dizziness, nausea, and pain in their limbs[6][7], which are the main causes for the Korean students to visit school nurses[8]. In this study, the most frequent cause for the students to visit school nurses was wounds $(34.1 \%)$, followed by abdominal pain $(30.7 \%)$ and headache $(17.6 \%)$. The key causes of headache included irregular sleep pattern and empty stomach $(36.9 \%)$, as well as relationship issues (30.1\%). The previous study by Park et al.[14] reported that the mean score 
for headache was highest $(2.26 \pm 1.26)$ among different physical symptoms experienced by adolescents. Moreover, Jung et al.[15] showed that, within their study period (1 year), headache was the most frequent symptom in Korean students that led to the school nurse visit. Rho et al.[9][11] demonstrated that $29 \%$ of the Korean students assessed in their study experienced repetitive headache[9], and repetitive or persistent headache forces the students to take time off from their study, hindering with their academic life both in school and after school[11]. Another study by Park et al.[14] reported that headache may affect the student's academic achievement, memory, and social relationship, and therefore the school nurses should carefully evaluate symptoms (i.e. migraine, psychological factors, and stress) that can bring negative effects onto headache[14]. More specifically, students who frequently visited school nurses due to headache, have severe headache, have migraine, or have had headache for a long-term in the past are more difficult to manage. In addition, since possible disturbances or dysfunctions from headache may hinder with normal school activities, the school nurses should assess the students' medical history and provide appropriate management[11].

Although the key responsibilities of school nurses include primary care in emergency situations and management of health-related issues, these health issues in students are not described in a standardized nursing terminology. Since diagnosis is made based on the students' description of their symptoms, it is difficult to efficiently manage headaches - which is one of the most common complaints of the students[7]. In this study, the daily mean proportion of students visiting school nurses due to headache was $21.2 \pm 13.5 \%$. Despite the fact that headache is one of the most common symptoms for the students to visit school nurses, only $12.5 \%$ of the school nurses in this study have had training lecture for management of headache in the past 3 years and only $9.7 \%$ of the participants were confident in the management of headache. The participants responded that standardized guideline for headache management $(49.4 \%)$ as well as training lecture $(43.8 \%)$ are needed. These findings suggest that the school nurses had limited opportunity to gain knowledge on the management of headache, and it is difficult to manage headaches with the current guideline that does not describe the management of headache in detail.

The previous study suggesting that headaches in adolescent and teenage students are heavily affected by temperamental characteristics and social relationship[16]. But there has not been a study that assessed whether or not the school nurses who manage these students are aware of the newest information and apply it to improve the performance of headache management. In order for valid and efficient performance, the person in charge - school nurses in this case should have perception of what needs to be performed[17]. For appropriate management of 
headache in the students, lack of knowledge and experience in the school nurses should be primarily considered. Although the scores for perception and performance of headache management in the cohort of school nurses in this study were above average $(3.88 \pm 0.33$ and $3.79 \pm 0.40$ respectively), the score for performance was relatively lower than the score for perception. Furthermore, there was a positive correlation between the perception and performance of headache management. The previous study by Jeon et al.[7] demonstrated similar pattern of scores for perception and performance of abdominal pain management (4.02 \pm 0.43 and $3.47 \pm 0.41$ respectively) and same positive correlation between the perception and performance. Therefore, if the school nurses have better awareness of common symptoms of the students, performance for management of those symptoms are also likely to improve. The observation that the score for performance was lower than the score for perception can be explained by the fact that school nurses are aware of the importance of headache management, but do not know the efficient treatment method.

With $87.5 \%$ of the participants responding that they have not had training lecture in the past 3 years, it is thought that the school nurses were given limited opportunity to obtain the most recent knowledge on the management of headache. On the contrary, $75.0 \%$ of the participants were interested in participating in the future training lecture, suggesting that the school nurses are interested in learning about headache management but is given limited opportunity due to several obstacles (i.e. practical work issue or insufficient number of programs offered). Therefore, additional training program should be provided to the school nurses so that they can obtain newest information on headache management and apply it in the actual performance. Moreover, to improve the performance of headache management for the school nurses, a standardized guideline describing the symptoms, intervention, and management of headache should be established.

Despite these interesting findings, there are a few limitations in this study. First, the participants of this study were only selected from the city of Gwangju, and therefore cannot represent the school nurse population in Korea. Second, although the training on headache management was provided after 1st survey, the response for 2nd survey was not obtained. Therefore, the changes in perception and performance after the training could not be assessed. Future studies should include school nurses from other regions, and assess the change in perception and performance after providing training by asking the participants to complete 2nd survey after the training. 


\section{Conclusion and Suggestions}

This study is a descriptive research study which is attempted to investigate the perceptions and performances of school nurses' headache management and identify factors affecting their performance in order to effectively manage headaches, which are the major health problems of children and adolescents.

In this study, a total of 176 data were analyzed by distributing a questionnaire to school nurses who participated in the training process of the $G$ metropolitan office of education. As a result of this study, school nurses' perception of headache management (range 1 to 5 points) averaged $3.88 \pm 0.33$ points, and the average for performing headache management (range 1 to 5 points) was $3.79 \pm 0.40$ points, so it was found that the actual performance was somewhat lower than the recognition level. And the relationship between the subject's perception of headache management and performance was statistically significant, showing a statistically significant correlation $(\mathrm{r}=0.50, \mathrm{P}<.001)$, so the higher the perception level, the higher the performance. As a result of multiple regression analysis, the factors affecting the performance of the school nurses's headache pain management were perception of headache management $(\beta$ $=$ ).500), and these factors were accounted for $24.5 \%$ of the headache management performance.

As a result, it is necessary to improve the perception of school nurses' headache management so that more efficient headache management can be achieved. In addition, school nurses believe that it is necessary to develop standardized guidelines to manage headaches effectively, so I would like to suggest the following.

First, since this study is targeted at school nurses located in G metropolitan city, it is difficult to generalize the results of the study, so it is necessary to expand the research to school nurses nationwide. Second, it is necessary to develop various training programs for effective headache management of school nurses. Third, on the basis of the latest evidence, it is suggested to develop programs and standardized guidelines to manage health problems, including headaches among children and adolescents.

\section{Acknowledgement}

"Written consent was obtained from the patient or their relative for publication of study". This study was supported by research fund from Chosun University Hospital, 2017 This study has not been reported previously 


\section{References}

[1] J. I. Um, S. E. Yi, The Epidemiological Characteristics of Health Problems of Middle School Students Based on School Health Clinic Service, The Journal of The Korean Society of School Health, (2007), Vol.20, No.1, pp.25-35. UCI : G704-001960.2007.20.1.007

[2] P. Guilday, School Nursing Practice Today: Implications for the Future, Journal of School Nursing, (2000), Vol.16, No.5, pp.25-31. DOI: 10.1177/105984050001600504

[3] J. H. Kim, H. S. So, Utilization of school health room and health problems among elementary and secondary school students, and nursing performance of school health teachers in a city, The Journal of Korea Contents Association, (2016), Vol.16, No.5, pp.180-193. DOI: 10.5392/JKCA.2016.16.05.180

[4] S. H. Hong, Usage and its trend of health clinic among middle school students (dissertation), Seoul : Univ. of Korea, Master's Thesis, (2013)

[5] J. O. Kwon, J. Oh, E. H. Kim, D. D. Hahn, Professional identity of elementary school health teachers: a grounded theory approach, Child Health Nursing Research, (2015), Vol.21, No.1, pp.64-73. DOI : 10.4094/chnr.2015.21.1.64

[6] J. Garber, L. S. Walker, J. Zemma, Somatization symptoms in a community sample of children and adolescents: Further validation of the Children's Somatization Inventory, Psychological Assessment, (1991), Vol.3, No.4, pp.588-595. DOI: 10.1037/1040-3590.3.4.588

[7] J. H. Jeon, S. K. Hwang, A Study on Perception and Performance of Abdominal Pain Management among Elementary School Health Teachers, Journal of the Korean Society of School Health, (2012), Vol.25, No.2, pp.222-230. UCI : G704-001960.2012.25.2.003

[8] J. H. Lee, H. A. Park, Development of a nursing care decision support system for health problems of the elementary and secondary school students, Healthcare Informatics Research, (2009), Vol.15, No.1, pp.71-81. UCI : G704-001070.2009.15.1.003

[9] Y. I. Rho, H. J. Chung, K. H. Lee, B. L. Eun, S. H. Eun, S. O. Nam, W. S. Kim, Y. O. Kim, H. J. Park, H. S. Kim, Prevalence and Clinical Characteristics of Primary Headaches Among School Children in South Korea: A Nationwide Survey, Headache: The Journal of Head and Face Pain, (2012), Vol.52, No.4, pp.592-599. DOI: $10.1111 / j .1526-4610.2011 .02001 . x$

[10] J. Y. Ahn, Y. I. Rho, Headache-related disability and predictor of disability in children and adolescents, Journal of the Korean Child Neurology Society, (2015), Vol.23, No.3, pp.104-110. DOI: 10.26815/jkcns.2015.23.3.104

[11] Y. I. Rho, J. Y. Joo, H. J. Chung, K. H. Lee, B. L. Eun, S. H. Eun, S. O. Nam, W. S. Kim, Y. O. Kim, Characterization and Predictors of Headache-Related Disability among School Children: A Population-Based Study, Journal of the Korean Child Neurology Society, (2017), Vol.25, No.1, pp.27-33. DOI : $10.26815 / \mathrm{jkcns} .2017 .25 .1 .27$

[12] R. E. Behrman, R. kliegman, Nelson textbook of pediatrics, 15th edition, WB Saunders Company, (1995)

[13] S. Y. Park, Y. I. Rho, School Nurses's Management for Schoolchildren with Headache, Journal of the Korean Child Neurology Society, (2017), Vol.25, No.1, pp.22-26. DOI : 10.26815/jkcns.2017.25.1.22 
The Relationship between the Perception and Performance of Headache Management among School Nurses

[14] Y. J. Park, Y. J. Im, Influence of Perceived Attachment Security and Social Support on Somatic Symptoms in Late School-Aged Children Using a School Health Clinic, Child Health Nursing Research, (2016), Vol.22, No.4, pp.370-378. DOI : 10.4094/chnr.2016.22.4.370

[15] M. S. Jung, Comparison of the Health Condition and the Satisfaction Level with School Life of the Male and Female Elementary School Pupils Utilizing the Clinics at School, Ajou University, Master's Thesis, (2003)

[16] H. K. Shin, The Relationship of Academic Stress, Negative Affectivity, Alexithymia, and Perceived Parenting Behavior to Somatization in Adolescents, Korean Journal of Clinical Psychology, (2002), Vol.21, No.1, pp.171-187. UCI : G704-000007.2002.21.1.014

[17] T. R. Sarbin, V. L. Allen, Role theory. In G. Lindzey \& E. Aronson (Eds.), The handbook of social psychology, (1968) 